\author{
a report by \\ John D Williams and H Feidhlim Woods
}

Professor of Nephrology and Head, Department of Nephrology, School of Medicine, Cardiff University, and

Senior Vice-President Medical, Fresenius Medical Care Asia-Pacific DOI:10.17925/EE.2006.00.02.29

Insulin resistance and the metabolic syndrome (MeS or syndrome $\mathrm{X}$ ) are common in patients with chronic kidney disease (CKD). Moreover, the presence of $\mathrm{MeS}$ is an independent predictor of subsequent development of CKD, and $\mathrm{MeS}$ in patients with CKD predicts subsequent cardiovascular events and cardiovascular mortality, as is the case in the general population.

In a recent analysis of the Atherosclerosis Risk in Communities Study in a total population of 10,096 non-diabetic subjects, the relative risk of developing CKD within a nine-year follow-up was 1.43 (95\% confidence interval (CI): 1.18-1.73) for patients with $\mathrm{MeS}$, and the increase in risk of CKD correlated with the number of components of the MeS and with increasing insulin resistance (assessed by homeostasis model assessment insulin resistance (HOMA-IR)).

Data from the Third National Health and Nutrition Examination (NHANES III) showed a prevalence of $\mathrm{MeS}$ of $18.8 \%$ in patients with normal glomerular filtration rate (GFR: $\left.>90 \mathrm{ml} / \mathrm{min} / 1.73 \mathrm{~m}^{2}\right), 21 \%$ in patients with mild renal impairment (GFR: $45-89 \mathrm{ml} / \mathrm{min} / 1.73 \mathrm{~m}^{2}$ ) and $33 \%$ in moderate CKD (GFR: $36-38 \mathrm{ml} / \mathrm{min} / 1.73 \mathrm{~m}^{2}$ ). Insulin resistance detected by various means, including impaired glucose tolerance, HOMA-IR and the euglycaemic hyperinsulinaemic glucose clamp (EGC) has been identified in approximately $30 \%$ of patients treated with haemodialysis (HD) or peritoneal dialysis (PD).

The cause of insulin resistance in CKD is almost certainly multifactorial. Initiation of dialysis treatment, whether by HD or PD, improves but does not normalise insulin sensitivity as measured by the EGC, suggesting that insulin resistance might be caused by a retained uraemic toxin. Protein carbamylation, increased in uraemia via the ureaisocyanate pathway, has also been suggested as a possible cause of insulin resistance. Other possible mechanisms include uraemic metabolic acidosis and disorders of intracellular ionic homeostasis.

In the past decade, many avenues of research have implicated inflammation and central adiposity in the aetiology of MeS. Visceral adipose tissue is a potent source of pro-inflammatory cytokines, and inflammatory cytokines - e.g. tumour necrosis factor alpha (TNF- $\alpha$ ) - reduce tissue sensitivity to insulin. Chronic low-grade inflammation as detected by minimally elevated c-reactive protein (CRP, $>3 \mathrm{mg} /$ litre) is common in patients with CKD and dialysis patients and, as with the general population, inflammation independently predicts cardiovascular events and mortality in renal patients. Interestingly, the number of components of MeS correlates in CKD with CRP at all levels of renal function. It is not yet clear whether inflammation and $\mathrm{MeS}$ are independent or interdependent risk factors for cardiovascular disease in CKD. Due to the fact that elucidation of the nature of any link between inflammation and $\mathrm{MeS}$ would be critical to appropriate management, this should be the subject of further study.

Recent research has also identified major disturbances of the endocrine functions of adipose tissue and of insulin-glycaemia-lipidaemia homeostasis in patients with renal failure. Patients with CKD have hyperleptinaemia and hyperadiponectinaemia with paradoxical energy-nutrition balance. It is debatable whether hyperleptinaemia is the major cause of the anorexia frequently observed in patients with advanced CKD, but the evidence increasingly points in that direction. Although CKD is associated with hyperadiponectinaemia, the cardiovascular protective effects of adiponectin seem to be intact in that lower levels of adiponectin increase the risk of cardiovascular events in CKD.

On-going research is likely to provide further insight into the complex and multisystem disorders of energy-nutrition homeostasis in CKD and provide direction for appropriate therapeutic intervention.

\section{Peritoneal Dialysis}

In this highly complex setting, CKD patients treated with PD deserve special attention. To achieve osmotic removal of retained fluid, PD fluids (PDFs) contain supraphysiological concentration of dextrose (1.5-4.25\% weight/volume, approximately 80-250mmol/litre), solutions of higher concentration

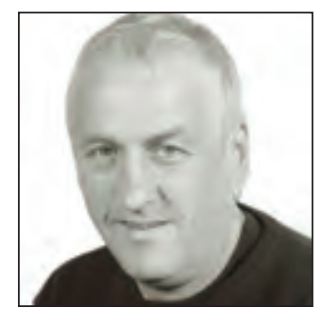

John D Williams

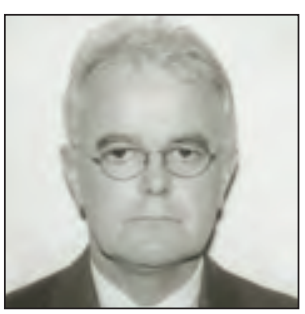

H Feidhlim Woods

John D Williams is Professor of Nephrology and Head of the Department of Nephrology at the School of Medicine, Cardiff University. This is the largest kidney research group in the UK and is internationally at the forefront of renal research. Professor Williams has lectured worldwide, is a member of numerous international editorial boards and has served on the organising committees of numerous international congresses. He has been a visiting professor in Australia, New Zealand, Japan, Taiwan, Poland and many other countries. Professor Williams also has a keen interest in sports medicine and is the physician to the Welsh National Rugby Team.

H Feidhlim Woods is Senior VicePresident of the Asia-Pacific region of Fresenius Medical Care. He was previously Managing Director for the same region, having joined the company in 1997 as Medical Director for the South Asia-Pacific region. Prior to that, Dr Woods was Medical Director of the National Kidney Foundation. He also spent 10 years in the Middle East, where he established nephrology dialysis programmes in Kuwait and the Sultanate of Oman. 
being prescribed to remove more fluid in an effort to control hypervolaemia and hypertension. Over time and associated with progressive loss of diuresis from the failing native kidneys, the average dextrose concentration of the PD prescription tends to rise. Depending on the period of exposure of the peritoneal cavity to the PDFs (the dwell time), up to $80 \%$ of the PDF glucose is absorbed and the $100-300 \mathrm{~g}$ glucose absorbed contributes $14-34 \%$ of daily energy intake.

The potential for this glucose load to cause problems such as obesity and hyperlipidaemia has long been discussed in the nephrology literature and alternatives to glucose for osmosis have been suggested and are commercially available. However, glucose remains the most commonly used and lowest cost osmotic agent. The question is whether this is a cause for concern.

\section{Obesity and Hyperlipidaemia}

There is no evidence that patients treated with PD (and thus receiving additional glucose loading) are more obese than patients treated with HD (and not glucose-loaded). More importantly, obesity in dialysis patients is not associated with increased risk of cardiovascular disease, as it is in the general there was a trend towards better patient survival in those with the highest peritoneal glucose loading.

Patterns of Dyslipidaemia

The typical pattern of hyperlipidaemia in patients with CKD is the association of moderate elevation of triglyceride levels and low or low to normal highdensity lipoprotein (HDL) cholesterol and/or a high total/HDL cholesterol ratio (dyslipidaemic features that also typify $\mathrm{MeS}$ ). Other features of uraemic dyslipidaemia include high lipoprotein (a) levels and an increase in small dense lipoproteins. This pattern persists essentially unchanged in HD patients.

In PD patients, the dyslipidaemia tends to worsen with a further rise in triglyceride levels and the emergence of hypercholesterolaemia. It might seem intuitive that these changes somehow relate to PDF glucose loading, but this could not be confirmed in cross-sectional or longitudinal studies. However, worsening dyslipidaemia has been related to impaired glucose tolerance and insulin resistance.

In one study, hypertriglyceridaemia was, after one year of PD treatment, confined to the approximate one-third of patients with insulin resistance as determined by the ratio of area under the curve

\section{Obesity in dialysis patients is not associated with increased risk of cardiovascular disease, as it is in the general population.}

population. In fact, 'reverse epidemiology' applies and obesity is associated with improved survival in dialysis patients, whether treated by PD or HD, but the protective effect of increased body mass index (BMI) is greater in HD patients.

On the other hand, low BMI and other indices of malnutrition are powerful, independent predictors of mortality in dialysis patients and the challenge for management of CKD is to achieve adequate protein energy intake. The daily calorie intake recommended for optimum protein energy nutrition in dialysis patients is 35 kilo calories (kcal) per kilo per day. This is not achieved by oral intake in many dialysis patients, which is why PDFderived glucose is an important source of calories in PD patients. Moreover, higher peritoneal calorie absorption does not inhibit oral calorie intake and, in at least one longitudinal survey of PD patients,
(AUC) insulin to AUC for glucose (AUC $\mathrm{ins}$ / $\left.A U C_{\text {glu }}\right)$. The remaining two-thirds of patients with normal glucose tolerance had no worsening of dyslipidaemia despite equivalent peritoneal glucose loading. Moreover, in a randomised controlled trial comparing a PD regimen with standard $(125 \pm 41 \mathrm{~g} /$ day to $159 \pm 40 \mathrm{~g} /$ day $)$ versus lower $(87 \pm 76 \mathrm{~g} /$ day to $111 \pm 76 \mathrm{~g} /$ day) peritoneal glucose load, six months of treatment with the lower glucose regimen was not associated with significant improvement in dyslipidaemia.

In another study, where one daily exchange of glucose PDFs was replaced with an exchange of an amino acid (AA) PDF, there was a modest fall in total cholesterol, triglycerides and free fatty acids after eight weeks of AA prescription. In that study, eight of the 20 patients also underwent two months of treatment with an even lower glucose regimen. 
In this subset, no changes in lipids were seen, which is consistent with the lack of effect described by Le Poole et al.

Insulin Sensitivity and

Glycaemia Homeostasis

Type 2 diabetes mellitus is now the most common cause and associated co-morbidity in dialysis patients. Additionally, as described above, insulin resistance is known to occur in non-diabetic uraemic patients and is only partially corrected by dialysis treatment. That $\mathrm{HD}$ and $\mathrm{PD}$ are equivalently effective in partial reversal of insulin resistance is inconsistent with a uniformly adverse effect of PDF glucose loading on insulin sensitivity.

On the other hand, it has been suggested that PD and the associated glucose loading induce insulin resistance. The problem with such studies is that they fail to take into account the insulin resistance that is present before dialysis is initiated.

In a single study of a small number of patients, HOMA-IR was reported to improve when one glucose exchange was replaced with a PDF containing the polyglucose icodextrin (from which calorie absorption is approximately $40 \%$ less than from a glucose exchange). However, the interpretation of the HOMA-IR result is questionable, as the patients were not in a steady state for glucose absorption (a prerequisite for use of HOMA-IR as measure of insulin resistance).

No Improved Glycaemic Control

Martikainen et al. were unable to show any improvement in insulin sensitivity in diabetic and non-diabetic patients after eight weeks of treatment where one glucose exchange was replaced with AA or icodextrin, and neither AA, nor icodextrin, nor a regimen where both solutions replaced two glucose exchanges improved glycaemic management (glycosylated haemoglobin percentage (HBA1c\%)) in diabetics. Replacement of a dextrose exchange with icodextrin also failed to improve glycaemic control over a period of one year in a small group of Canadian diabetic patients.

Hithaishi et al. were unable to report any improvement in glycaemic control in eight diabetic patients treated for three to 12 months with icodextrin. In five patients who completed a full 12 months of follow-up, the mean HBA $1 \mathrm{c} \%$ actually rose from $6.9 \pm 1.4 \%$ to $8.0 \pm 0.7 \%$.

\section{Improving survival}

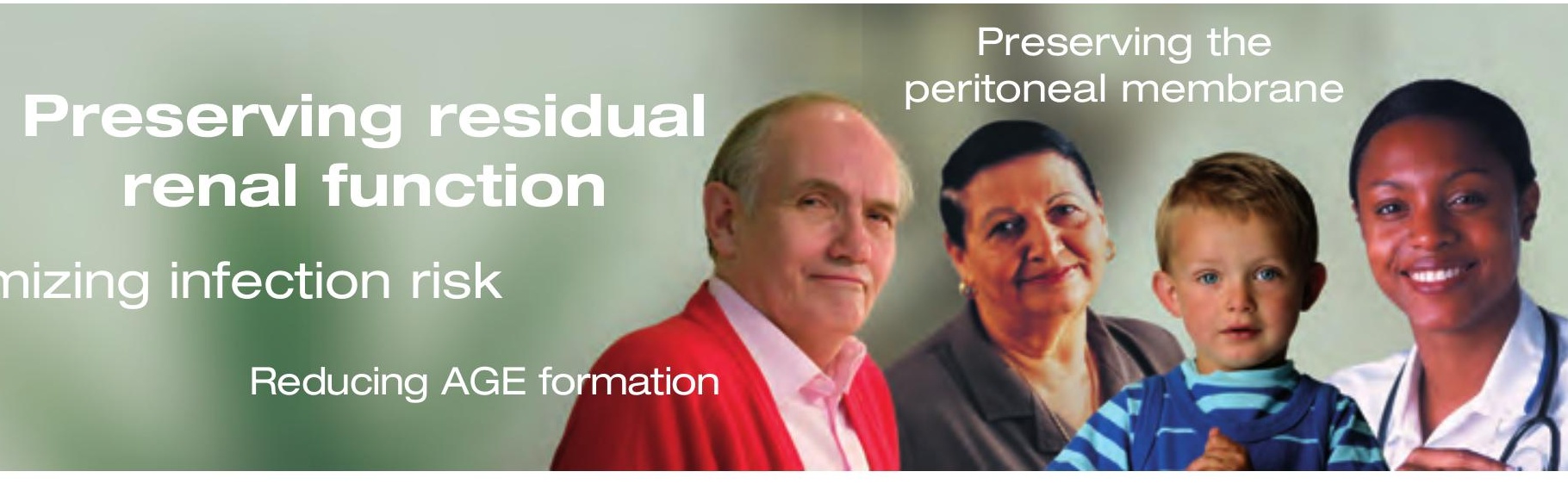

Fresenius Medical Care has focused continuous developments on improving dialysis therapy. In PD this has resulted in extraordinary new PD solutions, systems and services.

These intensive investments in R\&D are reflected in superior clinical results indicating better safety and improved survival.

Contact your local Fresenius Medical Care representative to find out more.

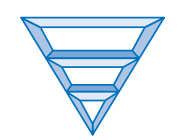

\section{Fresenius Medical Care}


On the other hand, Johnson et al. reported a fall in HBA1c from from $8.9 \pm 0.7 \%$ to $7.9 \pm 0.7 \%$ in 12 diabetics after three months of icodextrin administration.

Using a continuous glucose monitoring system (CGMS), Marshall et al. reported significantly improved short-term (one week) glycaemic control in eight diabetics treated with a PD regimen low in glucose compared with a standard regime. Average daily glycaemia was lower and the amplitude of hyperglycaemic excursions was reduced.

It is not clear why such an improvement in glycaemic control, if maintained over longer time and in a less controlled setting, does not translate into a consistent and significant improvement in HBA1c\%. Reducing the glucose loading from the PD prescription has, at best, a modest effect on the insulin-glycaemia axis. Moreover, a wider use of these alternative PDFs may not be appropriate for all patients. Use of AA-based PDFs is associated with worsening of uraemia, increased acidosis and an increase in plasma homocysteine (due to the methionine content of the AA PDFs). Hyperhomocysteinaemia is associated with an increased risk of cardiovascular disease in CKD patients. Use of PDFs with icodextrin is also patients treated with rosiglitazone, and the same drug increases insulin sensitivity and improves endothelial function in recipients of a renal transplant. These encouraging preliminary results for increasing insulin sensitivity, reducing inflammation and improving indices of endothelial function in renal patients treated with glitazones need confirmation in larger, placebocontrolled trials.

\section{Conclusions}

To the epidemic of diabetes mellitus type 2 in CKD and dialysis patients must now be added the equally large problems of insulin resistance and MeS. Their association with increased risk of adverse cardiovascular events should prompt specific investigation of CKD and dialysis patients to identify the cohort at risk, which, on the basis of the available epidemiological data, probably amounts to not less than one-third of CKD patients.

Non-specific intervention (e.g. regular exercise and modification of diet) favourably modifies insulin sensitivity and newer pharmacotherapies, such as the glitazones are proving of benefit for management of insulin resistance and $\mathrm{MeS}$ in the general population.

\section{The association [of insulin resistance and MeS] with increased risk of adverse cardiovascular events should prompt specific investigation of CKD and dialysis patients to identify the cohort at risk ...}

associated with a relatively high incidence of allergic skin diseases, including exfoliative dermatitis and the absorption of various maltoses from the polyglucose, and their persistently high concentration of plasma leads to true hyponatraemia, which tends to be more severe in diabetic patients.

\section{Insulin-sensitising}

Agents

By contrast, insulin sensitivity (HOMA-IR and $\mathrm{AUC}_{\mathrm{ins}} / \mathrm{AUC}_{\mathrm{glu}}$ ) was significantly increased in nondiabetic PD patients treated for four weeks with $4 \mathrm{mg} /$ day of the thiazolidinedione, rosiglitazone and the improvement in insulin sensitivity persisted at 12 weeks.

Wong et al. reported reduction in insulin requirement and lower CRP levels in 52 diabetic PD
CKD and dialysis patients should also benefit from an active approach to management.

For PD patients, where exposure to glucosecontaining PDFs is innate to their treatment and alternative PDFs with lower glucose content have little or no beneficial effect on insulin sensitivity or the features of MeS and where additional cost and adverse side effects should limit their more widespread use, the need for specific insulinsensitising strategies is even more pressing.

Nephrologists responsible for the care of dialysis patients must learn from their endocrinologist brethren how best to manage what is obviously a highly complex metabolic dysfunction(s) of their patients.

$A$ version of this article, with references, can be found in the Reference Section on the website supporting this business briefing (www.touchbriefings.com). 